\title{
Simple Receiving Scheme Based on Balanced Detection for Half-Cycled SSB DD-OFDM Signal
}

\author{
Pengfei YANG $^{\mathrm{a}, 1}$, XueCHEN ${ }^{\mathrm{b}}$ \\ ${ }^{a}$ The 6th Research Institute of China Electronics Corporation, Beijing, 100083, China \\ ${ }^{\mathrm{b}}$ State Key Laboratory of Information Photonics \& Optical Communications, Beijing \\ University of Posts \& Telecom, Beijing, 100876, China
}

\begin{abstract}
In this letter, we proposed a simple balanced-detection reception scheme for the half-cycled single-sideband direct-detected optical orthogonal frequency division multiplexing (HSSB DD-OFDM) signal with decreased guard band. By employing this scheme, each entire OFDM symbol can be recovered perfectly, while the signal-to-signal beat interference (SSBI) can be eliminated, the guard band can be reduced greatly and the tolerance to phase noise induced inter-channel interference (PN-ICI) and potential benefit of low peak to average power (PAPR) are retained. The simulation results demonstrate that a 40 Gbps 16-QAM HSSB DD-OFDM signal was achieved successfully.
\end{abstract}

Keywords. optical OFDM, direct-detection, beat interference, guard band

\section{Introduction}

Optical orthogonal frequency division multiplexing (OFDM) has been proposed and intensely investigated due largely to its enhanced spectral efficiency (SE) and excellent immunity to transmission deteriorations such as chromatic dispersion (CD) and polarization-mode dispersion (PMD), and fine granularity for dynamic bandwidth allocation [1]. According to the configurations of optical receivers, the optical OFDM systems could be classified into two sorts: coherent optical OFDM (CO-OFDM) and direct-detected optical OFDM (DD-OFDM). Compared to CO-OFDM, DD-OFDM systems require a simpler and more cost-effective receiver since the desired OFDM signal is acquired by detecting the beat outcomes of the carrier and signal using a square-law photodiode (PD), and therefore DD-OFDM could be one of the promising candidates to economically supply high bandwidth in metropolitan area networks and long-reach passive optical network [2].

The single-sideband format (SSB-OFDM) is adopted to combat the immanent CD-induced power fading associated with double-sideband (DSB) OFDM and to improve the SE in DD-OFDM. Typically, SSB-OFDM signal will be influenced by inherent signal-to-signal beat interference (SSBI) after square-law PD detection in case

1 Corresponding Author; E-mail: yhpdf@126.com 
the bandwidth of guard band (GB) between the main carrier and OFDM signal is smaller than the OFDM signal [3]. As mentioned in [1, 4], the blank frequency GB between the main carrier and the data signal was proposed to protect the signal from being interfered by the SSBI, and thus the system SE will be reduced to as much as half. Many research efforts have been made to address this issue. In [5], authors proposed the turbo coding technique to combat the SSBI at the cost of SE and complexity of forward-error-correction decoding. In [3], an iterative algorithm for the SSBI cancellation at the receiver was proposed for the virtual SSB-OFDM system, which demands large carrier-to-signal power ratio (CSPR) and increases the computation complexity. Moreover, in [6], a SSBI cancellation reception is proposed and analyzed in the SSB-OFDM system with an optical filter and a balanced receiver. However, the receiver sensitivity is reduced in that half of the overall optical power is used to rebuild the SSBI without contribution to the received OFDM signal. Another scheme which was called interleaved OFDM also can effectively remove the impingement of SSBI by loading data only in odd subcarriers, while sacrificing half of the system SE. It's noted that the interleaved OFDM signal can acquire tolerance toward PN-ICI and the intrinsic defect of OFDM signal, i.e., high PAPR can be significantly ameliorated in interleaved OFDM scheme [7-10]. Further, in [11], based on the symmetry characteristic of the interleaved OFDM symbol in the time domain, the half-cycled DD-OFDM transmission and receiving scheme was proposed to fight the SSBI without SE sacrifice. Specifically, the second half of interleaved OFDM symbol is cut away during the transmission and recovered by reversing the corresponding first half after square-law detection and analog to digital conversion (ADC). However, the simple reception scheme for half-cycled OFDM signal in [11] cannot guarantee effective OFDM signal recovery especially when the system is configured with small CSPR. Actually, the CSPR is as high as $20 \mathrm{~dB}$ in the experimental setup of DSB half-cycled DD-OFDM transmission to maintain performance compared with the traditional interleaved OFDM signal [11].

In this letter, a simple receiving scheme for half-cycled SSB optical OFDM signal is proposed. The receiver consists of an optical interleaver (IL), a $2 \times 23 \mathrm{~dB}$ optical coupler (OC) and a balanced PD pair. Thanks to perfect restoration of the half-cycled interleaved OFDM signal, the SSBI can be eliminated completely; on the other hand, the GB just needs to meet the requirement for splitting the optical carrier and SSB OFDM signal, so the SE can be meliorated greatly by decreasing the GB. The simulation results of the 40 Gbps 16-QAM HSSB DD-OFDM signal with considerably small GB demonstrate that the proposed scheme efficiently receives the half-cycled OFDM signal and system performance catches up with SSB DD-OFDM systems with sufficient GB or interleaved subcarrier configuration.

\section{System Model}

The SSB-OFDM signal may be generated by different methods, such as optical intensity modulation of radio frequency OFDM(RF-OFDM) signal with optical SSB filtering, optical IQ modulation of the baseband OFDM signal and attaching of an optical carrier, etc [1-6]. Figure 1 illustrates a conceptual diagram of the HSSB DD-OFDM system combined with the proposed receiving scheme. The output of continuous wave laser diode (LD) is split into two paths. One path passes through an 
IQ modulator which is biased at null point and driven by the complex baseband OFDM signal. The other path simply provides the main optical carrier and is mixed with the modulated optical OFDM signal using a $3 \mathrm{~dB}$ coupler. The fiber length in both paths of the coupler is matched to eliminate the propagation delay between the two paths. Separate generation of the optical OFDM signal and the main optical carrier enables flexible adjustment of the CSPR and large dynamic range for the baseband signal. Moreover, only the positive part of the inverse fast Fourier transform (IFFT) is filled to realize SSB-OFDM modulation and a GB is placed between the main optical carrier and OFDM signal by nullifying some subcarriers.

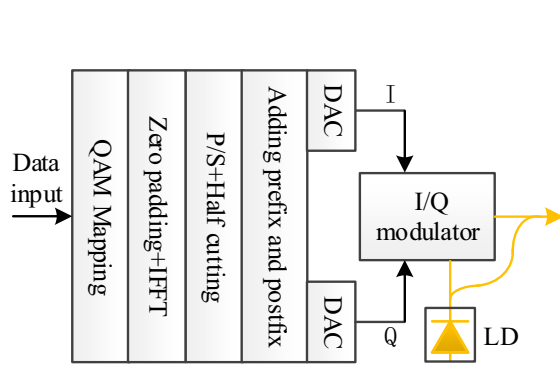

(a)

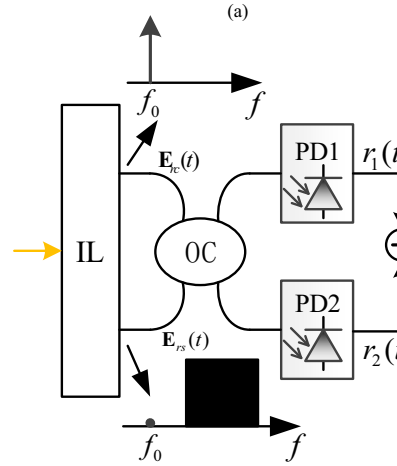

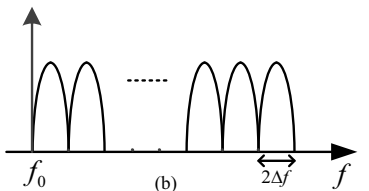

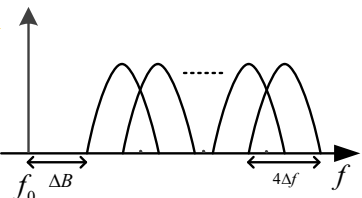

(c)

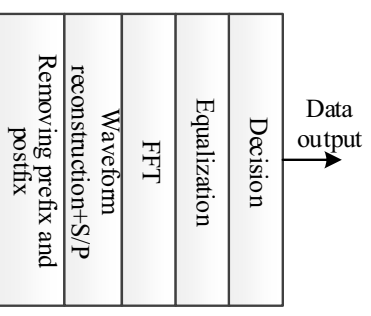

(d)

Figure 1. (a) HSSB DD-OFDM transmitter with optical I/Q modulation of the baseband OFDM signal and combination of a main optical carrier, (b) the spectral profile of conventional interleaved SSB DD-OFDM, (c) the spectral profile of HSSB DD-OFDM with a GB, (d) the proposed HSSB DD-OFDM receiver.

Let $X_{k}$ be the complex-valued symbol representing the constellation point on the $k$ th subcarrier for a given symbol. When subcarrier interleaving is introduced, symbols are only loaded in subcarriers with odd index and subcarriers with even index are loaded with " 0 "s. Then the IFFT realization of an interleaved OFDM signal for that symbol are given by $x_{m}$ where

$$
x_{m}=\frac{1}{N} \sum_{k=0}^{\frac{N}{2}-1} X_{2 k+1} e^{j \frac{2 \pi m(2 k+1)}{N}}, m=0,1,2, \ldots, N-1
$$

and $\mathrm{N}$ is the size of the IFFT. The time domain symmetry of the interleaved OFDM 
signal could be represented as

$$
x_{m+\frac{N}{2}}=\frac{1}{N} \sum_{k=0}^{\frac{N}{2}-1} X_{2 k+1} e^{j \frac{2 \pi\left(m+\frac{N}{2}\right)(2 k+1)}{N}}=-\frac{1}{N} \sum_{k=0}^{\frac{N}{2}-1} X_{2 k+1} e^{j \frac{2 \pi m(2 k+1)}{N}}=-x_{m}
$$

Eq. (2) demonstrates that the first and second halves of one interleaved OFDM symbol possess the opposite amplitude in time domain. In half-cycled interleaved OFDM scheme, the waveform of one symbol is truncated by only transmitting the first half. When the second half is somehow retrieved by the receiver, the complete waveform of that symbol can be reconstructed. By this means, the time taken to transfer data is halved and data rate is doubled. Therefore, the SE will remain unchanged despite the reserved subcarriers with even index. Meanwhile, to ensure the phase continuity between the recovered first and second halves in one symbol, the guard interval of the new half-cycled symbol should be carefully designed. As shown in Figure 2, except the routine prefix cloned from the tail of the interleaved OFDM symbol, the half-cycled OFDM symbol is added a postfix which is part of the head of the second half of the symbol.

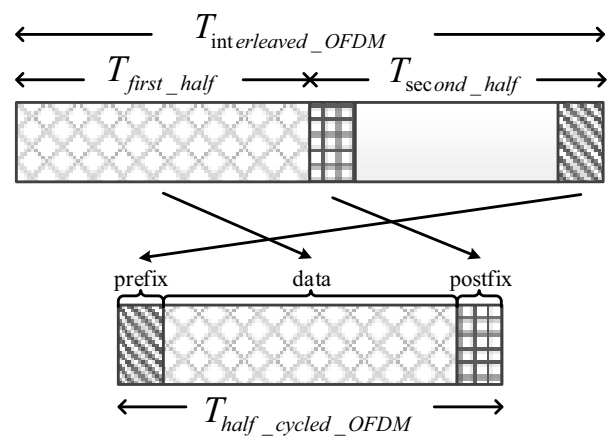

Figure 2. Half-cycled OFDM symbol with prefix and postfix.

As illustrated in Figure 1(c), the spectrum of the optical signal at the transmitter is the addition of a linear version of the electrical OFDM signal and a main optical carrier. Considering the truncating operation with OFDM symbol will not change the spectrum shape but broaden its spectral bandwidth by two subcarrier spacings, the transmitted optical HSSB DD-OFDM signal could be expressed as

$$
\mathbf{E}(t)=e^{j 2 \pi f_{0} t}+\beta e^{j 2 \pi\left(f_{0}+\Delta B\right) t} \sum_{k=0}^{\frac{N}{2}-1} X_{2 k+1} \Pi\left(t-\frac{T_{s}}{2}\right) e^{j 2 \pi f_{2 k+1} t}=\mathbf{E}_{\mathbf{c}}(t)+\mathbf{E}_{\mathbf{s}}(t),
$$

where $f_{0}$ is the main carrier frequency, $\Delta B$ is the GB between the main carrier frequency and OFDM signal, $\beta$ is the scaling coefficient that represents the CSPR; $\Pi(t)$ is the pulse shaping function, which is 1 in $\left[0, T_{s} / 2\right]$ and 0 otherwise; $T_{s}$ is 
the OFDM symbol duration. In Eq. (3), only one OFDM symbol is assumed for the sake of mathematical simplicity. If the fiber nonlinearity remains sufficiently low, the fiber channel can be modeled as a linear system. Therefore, the received optical signal at the IL still can be expressed in the form like Eq. (3). Then the IL separates the received optical HSSB DD-OFDM signal as the main optical carrier, $\mathbf{E}_{r c}(t)$, and the OFDM signal, $\mathbf{E}_{r s}(t)$. Note that the GB required by the IL could be much narrower than the bandwidth of OFDM signal. Then the main optical carrier and the OFDM signal are sent into a $2 \times 23 \mathrm{~dB}$ OC with the following transmission matrix:

$$
H=\left[\begin{array}{ll}
\frac{1}{2} & \frac{j}{2} \\
\frac{j}{2} & \frac{1}{2}
\end{array}\right] .
$$

In Eq. (4), we assumed that this ideal $3 \mathrm{~dB}$ OC has no additional loss and its two optical paths introduce the same time delay. Given that the two PDs have identical sensitivity, the two output photocurrents can be written as:

$$
\left[\begin{array}{c}
r_{1}(t) \\
r_{2}(t)
\end{array}\right]=\frac{1}{4}\left[\begin{array}{c}
\left|\mathbf{E}_{r c}(t)+j \mathbf{E}_{r s}(t)\right|^{2} \\
\left|j \mathbf{E}_{r c}(t)+\mathbf{E}_{r s}(t)\right|^{2}
\end{array}\right]=\frac{1}{4}\left[\begin{array}{l}
\left|\mathbf{E}_{r c}(t)\right|^{2}+\left|\mathbf{E}_{r s}(t)\right|^{2}+2 \operatorname{Re}\left\{j \mathbf{E}_{r c}^{*}(t) \mathbf{E}_{r s}(t)\right\} \\
\left|\mathbf{E}_{r c}(t)\right|^{2}+\left|\mathbf{E}_{r s}(t)\right|^{2}-2 \operatorname{Re}\left\{j \mathbf{E}_{r c}^{*}(t) \mathbf{E}_{r s}(t)\right\}
\end{array}\right] .
$$

For each photocurrent in Eq. (5), the first item is the direct current (DC) component; the second item is the so called SSBI which comes from the self-beating of the OFDM signal; and the last item is the desired carrier-signal beating products. By comparison, it's found that $r_{1}(t)$ and $r_{2}(t)$ actually correspond to the detected products of the first and second half of one interleaved OFDM symbol, respectively that is, the discarded second half of one interleaved OFDM symbol can be retrieved from $r_{2}(t)$. Meanwhile, regardless of the noise, due to the SSBI that could be strengthened by small CSPR configuration, the remained alternating current (AC) components (the second term and the third term) no longer strictly have the inverted amplitude after filtering the DC components in $r_{1}(t)$ and $r_{2}(t)$. So the restoration scheme for the half-cycled interleaved OFDM signal in [11] will be confined to the large CSPR condition. Further, via the subtraction operation we obtain

$$
r(t)=r_{1}(t)-r_{2}(t)=\operatorname{Re}\left\{j \mathbf{E}_{r c}^{*}(t) \mathbf{E}_{r s}(t)\right\}
$$

which is the desired signal included in the first half of one interleaved OFDM symbol. Let $S_{M S}(n)$ be the signal of $r(t)$ processed by sampling, timing and guard interval removing, then the final waveform of one symbol could be reconstructed as

$$
S(n)=\left[S_{M S}(n),-S_{M S}(n)\right]
$$


Although the first and the second halves of one symbol can be separately retrieved from $r_{1}(t)$ and $r_{2}(t)$ (hereafter, alternative scheme for short), one more analog-to-digital converter (ADC) and additional timing and guard interval removing operation will be required. Therefore, the proposed receiving scheme allows to simple and efficient recovery of the HSSB DD-OFDM signal while maintaining the immunity to SSBI interference.

\section{Results and Discussion}

A proof-of-concept simulation HSSB DD-OFDM link with 40 Gbps 16-QAM signal is built using VPItransmissionMaker and Matlab to investigate the feasibility of the proposed scheme.

The simulation platform's structure and detailed DSP for transmitter and receiver are described in Figure 1. The main system parameters are as follows: the FFT size is 256 while 60 interleaved subcarriers in the positive frequency bins are for the data; subcarriers between the first subcarriers and data-bearing subcarriers are reserved for GB varying from 1 to $10 \mathrm{GHz}$; overall length of prefix and postfix is 16 points; 20 training symbols are transmitted for overall channel estimation, followed by 500 data symbols. The baseband signal is modulated on the optical wave from an output branch of LD with central frequency of $193.1 \mathrm{THz}$ and linewidth of $100 \mathrm{MHz}$. The other output branch is used for optical carrier insertion. By adopting the carrier insertion, the signal can keep in linear modulation region when tweaking the CSPR by setting different split ratio of the LD's two branches. The HSSB DD-OFDM signal is transmitted through a $100 \mathrm{~km}$ standard single mode fiber with the power loss of 0.2 $\mathrm{dB} / \mathrm{km}$ and dispersion of $16 \mathrm{ps} / \mathrm{nm} \cdot \mathrm{km}$. An EDFA and tunable optical attenuator are installed before the receiver to regulate the optical power. In the receiver, the optical IL with sharp edge at $193.102 \mathrm{THz}$ splits the main optical carrier and optical OFDM signal. An ideal $2 \times 23 \mathrm{~dB}$ OC is used to combine the main optical carrier and the optical OFDM signal. Then the two mixed signals from the OC are sent into the balanced PD pair. The following DSP procedure including synchronization, guard interval removal, waveform reconstruction, FFT, channel estimation based on intra-symbol frequency domain averaging, one-tap equalization, 16-QAM de-mapping and BER/EVM computation.

Figure 3 illustrates the system performance in terms of BER as a function of the CSPR for the HSSB DD-OFDM signal received by two schemes. The CSPR of $\sim 0 \mathrm{~dB}$ is discovered to be the optimum value, which suggests that the best sensitivity is achieved while the carrier power equals to the signal power. Moreover, the performance difference between the two receiving schemes can be ignored. This optimum value agrees with the past known results of $0 \mathrm{~dB}$ for the gapped SSB DD-OFDM systems or interleaved SSB DD-OFDM systems, in which the desired signal and the SSBI noise are distributed over different frequency zones after the PD [7]. For a given total received optical power and fixed GB, as the CSPR deviates from the optimum value, the SNR decrease because the optical noise and transmission distortions become the leading factors. 


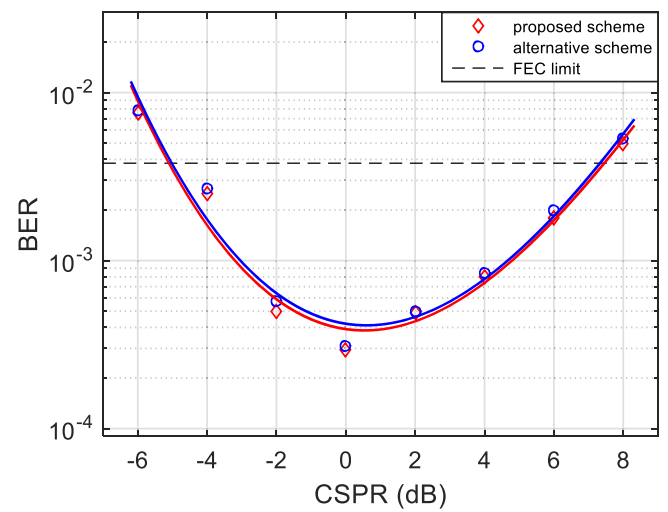

Figure 3. BER as the function of CSPR at the laser linewidth $=1 \mathrm{MHz}, \mathrm{GB}=5 \mathrm{GHz}$, received optical power $=-15 \mathrm{dBm}$.

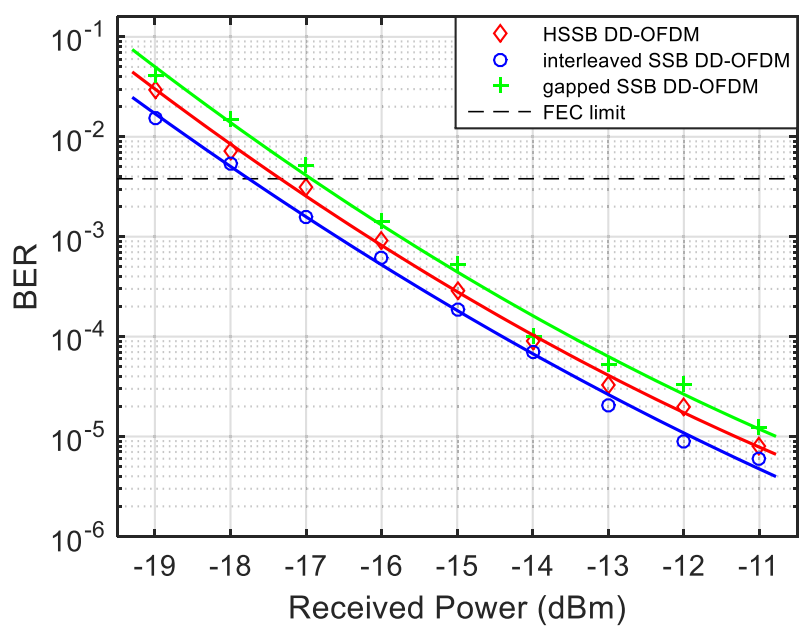

Figure 4. BER as the function of received optical power for HSSB DD-OFDM $(\mathrm{GB}=5 \mathrm{GHz})$, gapped SSB $\mathrm{DD}-\mathrm{OFDM}(\mathrm{GB}=10 \mathrm{GHz})$ and interleaved SSB DD-OFDM at the laser linewidth $=1 \mathrm{MHz}, \mathrm{CSPR}=0 \mathrm{~dB}$.

In Figure 4 the BER performance versus the received optical power is carried out for the proposed, the previous gapped SSB DD-OFDM system and interleaved SSB DD-OFDM system at $40 \mathrm{Gbps}$ with 16 QAM. The receiver sensitivities of HSSB DD-OFDM and interleaved SSB DD-OFDM are very close, which proves that HSSB DD-OFDM is practical. Compared to interleaved SSB DD-OFDM, HSSB DD-OFDM's receiver sensitivity penalty mainly comes from the synchronization timing error which affects the phase continuity between the recovered first and second halves in one symbol. Due to low PAPR and tolerance toward PN-ICI, HSSB DD-OFDM achieves slight better receiver sensitivity than gapped SSB DD-OFDM. 


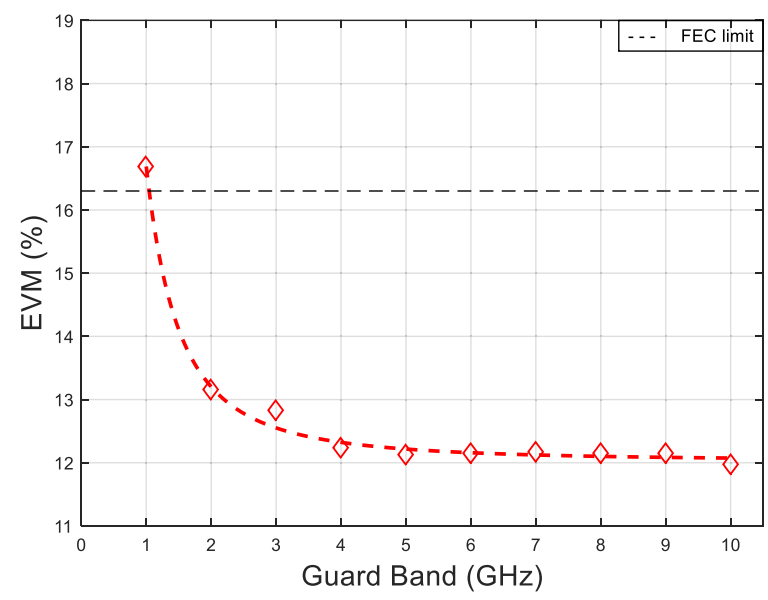

Figure 5. EVM as the function of GB with the laser linewidth $=1 \mathrm{MHz}$, received optical power $=-15 \mathrm{dBm}$.

To check the influence of GB on the HSSB DD-OFDM signal, the EVMs computed from the simulated symbols are in Figure 5. It's found that the EVMs are almost no change when the GB is equal to or bigger than $5 \mathrm{GHz}$. The EVM increases slowly and keeps below the FEC limit until the GB is reduced to $2 \mathrm{GHz}$, therefore the $\mathrm{SE}$ is meliorated greatly. As the GB is smaller than $2 \mathrm{GHz}$, the edge of the optical IL destroys the OFDM signal. Figure 6 gives the EVMs of the HSSB DD-OFDM signal with different laser linewidth from $1 \mathrm{MHz}$ to $10 \mathrm{MHz}$. It is found that EVM ascends almost linearly with the increment of laser linewidth and yet keeps below the FEC limit. Hence the HSSB DD-OFDM signal has good tolerance of laser linewidth.

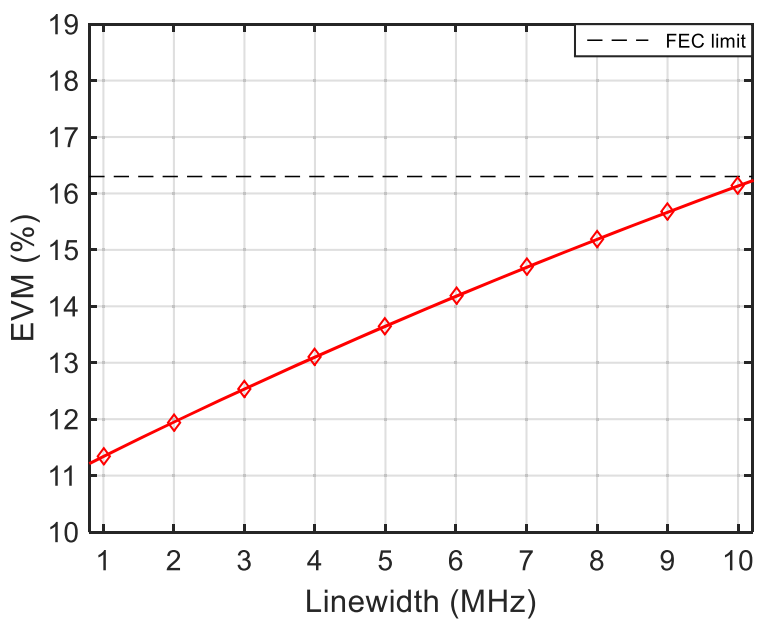

Figure 6. $\mathrm{EVM}$ as the function of the laser linewidth $=1 \mathrm{MHz}$, received optical power $=-15 \mathrm{dBm}$ and $\mathrm{GB}=5$ $\mathrm{GHz}$. 


\section{Conclusion}

A simple receiving scheme for half-cycled SSB DD-OFDM signal has been proposed and demonstrated. In this scheme, since the half-cycled interleaved OFDM signal can be well retrieved, the immunity to SSBI interference is retained with the greatly meliorated SE despite the increased complexity of the receiver. We investigated the system performance with 40 Gbps 16-QAM transmission simulations. The simulation results show that the proposed scheme possesses approximate performance compared with typical SSB DD-OFDM system and interleaved SSB DD-OFDM system, inherits low PAPR and tolerance toward PN-ICI from interleaved OFDM system and lowers the requirements for $\mathrm{GB}$ and laser linewidth.

\section{References}

[1] A. J. Lowery and J. Armstrong, "Orthogonal-frequency-division multiplexing for dispersion compensation of long-haul optical systems", Opt. Express, vol. 14, no. 6, pp. 2079 -2084, 2006.

[2] J. Armstrong, "OFDM for optical communications," J. Lightwave. Technol., vol. 27, no. 3, pp. 189204, Feb. 1, 2009.

[3] W.-R. Peng, et al., "Spectrally efficient direct detected OFDM transmission employing an iterative estimation and cancellation technique," Opt. Express, vol. 17, no. 11, pp. 9099-9111, 2009.

[4] W.-R. Peng, et al., "Spectrally Efficient Direct-Detected OFDM Transmission Incorporating a Tunable Frequency Gap and an Iterative Detection Techniques," J. Lightwave. Technol., vol. 27, pp. 5723-5735, 2009.

[5] Z. Cao, et al., "Direct-detection optical OFDM transmission system without frequency guard band," IEEE Photon. Technol. Lett., vol. 22, no. 11, 736-738, 2010.

[6] S. A. Nezamalhosseini, et al., "Theoretical and experimental investigation of direct detection optical OFDM transmission using beat interference cancellation receiver," Opt. Express, vol. 21, no. 13, pp. 15237-15246, 2013.

[7] W.-R. Peng, et al., "Theoretical and experimental investigations of direct-detected RF-tone-assisted optical OFDM systems,” J. Lightwave Technol., vol. 27, no. 10, pp. 1332-1339, 2009.

[8] Z. Cao, et al., "Interleaved and partial transmission interleaved optical coherent orthogonal frequency division multiplexing," Opt. Lett., vol. 39, no. 7, pp. 2179-2182, 2014.

[9] Mondal, M. R. H., and Panta, K., "Performance analysis of spatial OFDM for pixelated optical wireless systems.” Trans. Emerging Tel. Tech., vol. 28, SP. e2948, 2017.

[10] Bharati, S., and Podder, P., "Adaptive PAPR Reduction Scheme for OFDM Using SLM with the Fusion of Proposed Clipping and Filtering Technique in Order to Diminish PAPR and Signal Distortion." Wireless Pers. Commun., vol. 113, pp. 2271-2288, (2020).

[11] F. Li, et al., "SSMI cancellation in direct-detection optical OFDM with novel half-cycled OFDM," Opt. Express, vol. 21, no. 23, pp. 28543-28549, 2013. 